\title{
PFAFFIANS, HAFNIANS AND PRODUCTS OF REAL LINEAR FUNCTIONALS
}

\author{
PÉter E. Frenkel
}

\begin{abstract}
We prove pfaffian and hafnian versions of Lieb's inequalities on determinants and permanents of positive semi-definite matrices. We use the hafnian inequality to improve the lower bound of Révész and Sarantopoulos on the norm of a product of linear functionals on a real Euclidean space (this subject is sometimes called the 'real linear polarization constant' problem).
\end{abstract}

\section{-1. Introduction}

The contents of this paper are as follows. In Section 0, we sketch one part of the historic background: classical inequalities on determinants and permanents of positive semi-definite matrices. In Section 1, we prove pfaffian and hafnian versions of these inequalities, and we formulate Conjecture 1.5, another hafnian inequality. In Section 2, we apply the hafnian inequality of Theorem 1.4 to our main goal: improving the lower bound of Révész and Sarantopoulos on the norm of a product of linear functionals on a real Euclidean space (this subject is sometimes called the 'real linear polarization constant' problem, its history is sketched at the end of the paper). This is achieved in Theorem 2.3. We point out that Conjecture 1.5 would be sufficient to completely settle the real linear polarization constant problem.

\section{Old inequalities on determinants and permanents}

Recall that the determinant and the permanent of an $n \times n$ matrix $A=\left(a_{i, j}\right)$ are defined by

$$
\operatorname{det} A=\sum_{\pi \in \mathfrak{S}_{n}}(-1)^{\pi} \prod_{i=1}^{n} a_{i, \pi(i)}, \quad \quad \text { per } A=\sum_{\pi \in \mathfrak{S}_{n}} \prod_{i=1}^{n} a_{i, \pi(i)},
$$

where $\mathfrak{S}_{n}$ is the symmetric group on $n$ elements. Throughout this section, we assume that $A$ is a positive semi-definite Hermitian $n \times n$ matrix (we write $A \geq 0$ ). For such $A$, Hadamard proved that

$$
\operatorname{det} A \leq \prod_{i=1}^{n} a_{i, i}
$$

Received by the editors April 11, 2007.

2000 Mathematics Subject Classification. 46C05, 15A15.

Key words and phrases. polarization constant, real Euclidean space, hafnian, pfaffian, positive semi-definite matrix.

Partially supported by OTKA grants T 046365, K 61116, and NK 72523. 
with equality if and only if $A$ has a zero row or is a diagonal matrix. Fischer generalized this to

$$
\operatorname{det} A \leq \operatorname{det} A^{\prime} \cdot \operatorname{det} A^{\prime \prime}
$$

for

$$
A=\left(\begin{array}{cc}
A^{\prime} & B \\
B^{*} & A^{\prime \prime}
\end{array}\right) \geq 0
$$

with equality if and only if $\operatorname{det} A^{\prime} \cdot \operatorname{det} A^{\prime \prime} \cdot B=0$.

Concerning the permanent of a positive semi-definite matrix, Marcus [Mar1, Mar2] proved that

$$
\text { per } A \geq \prod_{i=1}^{n} a_{i, i}
$$

with equality if and only if $A$ has a zero row or is a diagonal matrix. Lieb [L] generalized this to

$$
\text { per } A \geq \operatorname{per} A^{\prime} \cdot \operatorname{per} A^{\prime \prime}
$$

for $A$ as in (1), with equality if and only if $A$ has a zero row or $B=0$. Moreover, he proved that in the polynomial $P(\lambda)$ of degree $n^{\prime}$ (=size of $A^{\prime}$ ) defined by

$$
P(\lambda)=\operatorname{per}\left(\begin{array}{cc}
\lambda A^{\prime} & B \\
B^{*} & A^{\prime \prime}
\end{array}\right)=\sum_{t=0}^{n^{\prime}} c_{t} \lambda^{t},
$$

all coefficients $c_{t}$ are real and non-negative. This is indeed a stronger theorem since it implies

$$
\text { per } A=P(1)=\sum_{t=0}^{n^{\prime}} c_{t} \geq c_{n^{\prime}}=\operatorname{per} A^{\prime} \cdot \operatorname{per} A^{\prime \prime} .
$$

Đoković [D, Mi] gave a simple proof of Lieb's inequalities, and showed also that if $A^{\prime}$ and $A^{\prime \prime}$ are positive definite then $c_{n^{\prime}-t}=0$ if and only if all subpermanents of $B$ of order $t$ vanish. Lieb [L] also states an analogous (and analogously provable) theorem for determinants: for $A$ as in (1), let

$$
D(\lambda)=\operatorname{det}\left(\begin{array}{cc}
\lambda A^{\prime} & B \\
B^{*} & A^{\prime \prime}
\end{array}\right)=\sum_{t=0}^{n^{\prime}} d_{t} \lambda^{t} .
$$

If $\operatorname{det} A^{\prime} \cdot \operatorname{det} A^{\prime \prime}=0$, then $D(\lambda)=0$. If $A^{\prime}$ and $A^{\prime \prime}$ are positive definite, then $(-1)^{t} d_{n^{\prime}-t}$ is positive for $t \leq \mathrm{rk} B$ and is zero for $t>\operatorname{rk} B$.

Remark. In all of Lieb's inequalities mentioned above, the condition that the matrix $A$ is positive semi-definite can be replaced by the weaker condition that the diagonal blocks $A^{\prime}$ and $A^{\prime \prime}$ are positive semi-definite. The proof goes through virtually unchanged. Alternatively, this stronger form of the inequalities can be easily deduced from the seemingly weaker form above. 


\section{New inequalities on pfaffians and hafnians}

For an $n \times n$ matrix $A=\left(a_{i, j}\right)$ and subsets $S, T$ of $N:=\{1, \ldots, n\}$, we write $A_{S, T}:=\left(a_{i, j}\right)_{i \in S, j \in T}$. If $|T|=2 t$ is even, we write

$$
(-1)^{T}:=(-1)^{t+\sum_{j \in T} j} .
$$

1.1. Pfaffians. As far as the applications in Section 2 are concerned, this subsection may be skipped.

Recall that the pfaffian of a $2 n \times 2 n$ antisymmetric matrix $C=\left(c_{i, j}\right)$ is defined by

$$
\text { pf } C=\frac{1}{n ! 2^{n}} \sum_{\pi \in \mathfrak{S}_{2 n}}(-1)^{\pi} c_{\pi(1), \pi(2)} \cdots c_{\pi(2 n-1), \pi(2 n)}
$$

We have $(\operatorname{pf} C)^{2}=\operatorname{det} C$.

For antisymmetric $A$ and symmetric $B$, both of size $n \times n$, we consider the polynomial

$$
(-1)^{\lfloor n / 2\rfloor} \text { pf }\left(\begin{array}{cc}
-\lambda A & B \\
-B & A
\end{array}\right)=\sum_{t=0}^{\lfloor n / 2\rfloor} p_{t} \lambda^{t} .
$$

Theorem 1.1. Let $A$ and $B$ be real $n \times n$ matrices with $A$ antisymmetric and $B$ symmetric. If $B$ is positive semi-definite, then $p_{t} \geq 0$ for all $t$. If $B$ is positive definite, then $p_{t}>0$ for $t \leq(\operatorname{rk} A) / 2$ and $p_{t}=0$ for $t>(\operatorname{rk} A) / 2$.

Proof. If $B=\left(b_{i, j}\right)$ is positive semi-definite, then there exist vectors $x_{1}, \ldots, x_{n}$ in a real Euclidean space $V$ such that $\left(x_{i}, x_{j}\right)=b_{i, j}$. Recall that in the exterior tensor algebra $\Lambda V$ a positive definite inner product (and the corresponding Euclidean norm) is defined by

$$
\left(\bigwedge v_{i}, \bigwedge w_{j}\right):=\operatorname{det}\left(\left(v_{i}, w_{j}\right)\right)
$$

We have

$$
\begin{array}{r}
p_{t}=\sum_{|S|=2 t} \sum_{|T|=2 t}(-1)^{S}(-1)^{T} \operatorname{pf} A_{S, S} \cdot \operatorname{pf} A_{T, T} \cdot \operatorname{det} B_{N \backslash S, N \backslash T}= \\
=\sum_{|S|=2 t} \sum_{|T|=2 t}\left((-1)^{S} \operatorname{pf} A_{S, S} \cdot \bigwedge_{i \notin S} x_{i},(-1)^{T} \operatorname{pf} A_{T, T} \cdot \bigwedge_{j \notin T} x_{j}\right)= \\
=\left|\sum_{|S|=2 t}(-1)^{S} \operatorname{pf} A_{S, S} \cdot \bigwedge_{i \notin S} x_{i}\right|^{2} \geq 0 .
\end{array}
$$

Assume that $B$ is positive definite. Then the vectors $x_{i}$ are linearly independent. It follows that the tensors $\bigwedge_{i \notin S} x_{i}$ are also linearly independent as $S$ runs over the subsets of $N$. Thus $p_{t}=0$ if and only if pf $A_{S, S}=0$ for all $|S|=2 t$, i.e., if and only if $2 t>\mathrm{rk} A$.

Theorem 1.2. Let $A$ and $B$ be real $n \times n$ matrices with $A$ antisymmetric and $B$ symmetric. Let $\lambda \geq 0$. If $B$ is positive semi-definite, then

$$
(-1)^{\lfloor n / 2\rfloor} \operatorname{pf}\left(\begin{array}{cc}
-\lambda A & B \\
-B & A
\end{array}\right) \geq \operatorname{det} B .
$$


If $B$ is positive definite, then equality occurs if and only if $\lambda A=0$.

Proof. The left hand side is

$$
p_{0}+p_{1} \lambda+\cdots+p_{\lfloor n / 2\rfloor} \lambda^{\lfloor n / 2\rfloor} .
$$

The right hand side is $p_{0}$.

I am grateful to the anonymous referee of this paper for the idea of the following alternative proof of Theorems 1.1 and 1.2. We may assume $B>0$, since every positive semi-definite matrix is a limit of positive definite ones. The matrix $B^{-1 / 2} A B^{-1 / 2}$ being real and antisymmetric, there exists a unitary matrix $U$ such that $D:=U^{-1} B^{-1 / 2} A B^{-1 / 2} U$ is diagonal with purely imaginary eigenvalues $a_{1} \sqrt{-1}$, $\ldots, a_{n} \sqrt{-1}$. The real multiset $\left\{a_{1}, \ldots, a_{n}\right\}$ is invariant under $a \leftrightarrow-a$. We have

$$
\begin{aligned}
\left(\sum p_{t} \lambda^{t}\right)^{2}= & \operatorname{det}\left(\begin{array}{cc}
-\lambda A & B \\
-B & A
\end{array}\right)=\operatorname{det}\left(\begin{array}{cc}
-\lambda \sqrt{B} U D U^{-1} \sqrt{B} & B \\
-B & \sqrt{B} U D U^{-1} \sqrt{B}
\end{array}\right)= \\
& =\operatorname{det}\left(\left(\begin{array}{cc}
\sqrt{B} U & 0 \\
0 & \sqrt{B} U
\end{array}\right)\left(\begin{array}{cc}
-\lambda D & \mathbf{1} \\
-\mathbf{1} & D
\end{array}\right)\left(\begin{array}{cc}
U^{-1} \sqrt{B} & 0 \\
0 & U^{-1} \sqrt{B}
\end{array}\right)\right)= \\
& =\operatorname{det} \sqrt{B}^{4} \cdot \prod_{i=1}^{n} \operatorname{det}\left(\begin{array}{cc}
-\lambda a_{i} \sqrt{-1} & 1 \\
-1 & a_{i} \sqrt{-1}
\end{array}\right)=\operatorname{det} B^{2} \cdot \prod_{i=1}^{n}\left(1+a_{i}^{2} \lambda\right) .
\end{aligned}
$$

Extracting square roots, and choosing the sign in accordance with $p_{0}=+\operatorname{det} B$, we get

$$
\sum p_{t} \lambda^{t}=(-1)^{\lfloor n / 2\rfloor} \operatorname{pf}\left(\begin{array}{cc}
-\lambda A & B \\
-B & A
\end{array}\right)=\operatorname{det} B \cdot \prod_{a_{i}>0}\left(1+a_{i}^{2} \lambda\right),
$$

whence both theorems immediately follow, since $\operatorname{det} B>0$.

1.2. Hafnians. Recall that the hafnian of a $2 n \times 2 n$ symmetric matrix $C=\left(c_{i, j}\right)$ is defined by

$$
\text { haf } C=\frac{1}{n ! 2^{n}} \sum_{\pi \in \mathfrak{S}_{2 n}} c_{\pi(1), \pi(2)} \cdots c_{\pi(2 n-1), \pi(2 n)} .
$$

For symmetric $A$ and $B$, both of size $n \times n$, we consider the polynomial

$$
\operatorname{haf}\left(\begin{array}{cc}
\lambda A & B \\
B & A
\end{array}\right)=\sum_{t=0}^{\lfloor n / 2\rfloor} h_{t} \lambda^{t} .
$$

Theorem 1.3. Let $A$ and $B$ be symmetric real $n \times n$ matrices. If $B$ is positive semidefinite, then $h_{t} \geq 0$ for all $t$. If $B$ is positive definite, then $h_{t}=0$ if and only if all $2 t \times 2 t$ subhafnians of $A$ vanish.

Proof. If $B=\left(b_{i, j}\right)$ is positive semi-definite, then there exist vectors $x_{1}, \ldots, x_{n}$ in a real Euclidean space $V$ such that $\left(x_{i}, x_{j}\right)=b_{i, j}$. Recall [Mar1, Mar2, MN, Mi] that in the symmetric tensor algebra $S V$ a positive definite inner product (and the corresponding Euclidean norm) is defined by

$$
\left(\prod v_{i}, \prod w_{j}\right):=\operatorname{per}\left(\left(v_{i}, w_{j}\right)\right) .
$$


We have

$$
\begin{array}{r}
h_{t}=\sum_{|S|=2 t} \sum_{|T|=2 t} \text { haf } A_{S, S} \cdot \operatorname{haf} A_{T, T} \cdot \operatorname{per} B_{N \backslash S, N \backslash T}= \\
=\left|\sum_{|S|=2 t} \operatorname{haf} A_{S, S} \cdot \prod_{i \notin S} x_{i}\right|^{2} \geq 0 .
\end{array}
$$

Assume that $B$ is positive definite. Then the vectors $x_{i}$ are linearly independent. It follows that the tensors $\prod_{i \notin S} x_{i}$ are also linearly independent as $S$ runs over the subsets of $N$. Thus $h_{t}=0$ if and only if haf $A_{S, S}=0$ for all $|S|=2 t$.

Theorem 1.4. Let $A$ and $B$ be symmetric real $n \times n$ matrices. Let $\lambda \geq 0$. If $B$ is positive semi-definite, then

$$
\operatorname{haf}\left(\begin{array}{cc}
\lambda A & B \\
B & A
\end{array}\right) \geq \operatorname{per} B
$$

If $B$ is positive definite, then equality occurs if and only if $A$ is a diagonal matrix or $\lambda=0$.

Proof. The left hand side is

$$
h_{0}+h_{1} \lambda+\cdots+h_{\lfloor n / 2\rfloor} \lambda^{\lfloor n / 2\rfloor} .
$$

The right hand side is $h_{0}$.

Setting $A=B$ and $\lambda=1$, and combining with Marcus's inequality (2), we arrive at case $p=1$ of

Conjecture 1.5. If $A=\left(a_{i, j}\right)$ is a positive semi-definite symmetric real $n \times n$ matrix, then the hafnian of the $2 p n \times 2 p n$ matrix consisting of $2 p \times 2 p$ blocks $A$ is at least $(2 p-1) ! !^{n} \prod a_{i, i}^{p}$, with equality if and only if $A$ has a zero row or is a diagonal matrix.

\section{Products of real linear functionals}

In this section, we apply Theorem 1.4 to products of jointly normal random variables and then to products of real linear functionals, which was the main motivation for this work. The ideas in this section are analogous to those that Arias-de-Reyna [A] used in the complex case.

Let $\xi_{1}, \ldots, \xi_{d}$ denote independent random variables with standard Gaussian distribution, i.e., with joint density function $(2 \pi)^{-d / 2} \exp \left(-|\xi|^{2} / 2\right)$, where $|\xi|^{2}=\sum \xi_{k}^{2}$. We write $E f(\xi)$ for the expectation of a function $f=f(\xi)=f\left(\xi_{1}, \ldots, \xi_{d}\right)$. Recall that

$$
E \xi_{k}^{2 p}=(2 p-1) ! !=(2 p-1)(2 p-3) \cdots 3 \cdot 1
$$

for $k=1, \ldots, d$ (easy inductive proof via integration by parts), and thus

$$
E \prod_{k=1}^{d} \xi_{k}^{2 p_{k}}=\prod_{k=1}^{d}\left(2 p_{k}-1\right) ! !
$$

On $\mathbb{R}^{d}$, we write $(\cdot, \cdot)$ for the standard Euclidean inner product. We recall the well-known [B2, G, S, Z] 
Wick formula. Let $x_{1}, \ldots, x_{n}$ be vectors in $\mathbb{R}^{d}$ with Gram matrix $A=\left(\left(x_{i}, x_{j}\right)\right)$. Then

$$
E \prod_{i=1}^{n}\left(x_{i}, \xi\right)=\operatorname{haf} A .
$$

(For odd $n$, we define haf $A=0$.)

Proof. Both sides are multilinear in the $x_{i}$, so we may assume that each $x_{i}$ is an element of the standard orthonormal basis $e_{1}, \ldots, e_{d}$. If there is an $e_{k}$ that occurs an odd number of times among the $x_{i}$, then both sides are zero. If each $e_{k}$ occurs $2 p_{k}$ times, then the left hand side is $E \prod_{k=1}^{d} \xi_{k}^{2 p_{k}}$, and the right hand side is $\prod_{k=1}^{d}\left(2 p_{k}-\right.$ $1) ! !$, which are equal.

The following theorems are easy corollaries of Theorem 1.4 together with the Wick formula (4) and Marcus's theorem (2).

Theorem 2.1. If $X_{1}, \ldots, X_{n}$ are jointly normal random variables with zero expectation, then

$$
E\left(X_{1}^{2} \cdots X_{n}^{2}\right) \geq E X_{1}^{2} \cdots E X_{n}^{2} .
$$

Equality holds if and only if they are independent or at least one of them is almost surely zero.

Proof. The variables can be written as $X_{i}=\left(x_{i}, \xi\right)$ with $\xi$ of standard normal distribution and the $x_{i}$ constant vectors with a positive semi-definite Gram matrix $A=\left(a_{i, j}\right)=\left(\left(x_{i}, x_{j}\right)\right)$. Then

$$
\begin{array}{r}
E \prod_{i=1}^{n} X_{i}^{2}=E \prod_{i=1}^{n}\left(x_{i}, \xi\right)^{2}= \\
=\operatorname{haf}\left(\begin{array}{cc}
A & A \\
A & A
\end{array}\right) \geq \operatorname{per} A \geq \prod_{i=1}^{n} a_{i, i}= \\
=\prod_{i=1}^{n} E\left(x_{i}, \xi\right)^{2}=\prod_{i=1}^{n} E X_{i}^{2},
\end{array}
$$

with equality if and only if $A$ is a diagonal matrix or has a zero row, i.e., the $x_{i}$ are pairwise orthogonal or at least one of them is zero.

The generalization of Theorem 2.1 to an arbitrary even exponent $2 p$ is equivalent to Conjecture 1.5.

Theorem 2.2. For any $x_{1}, \ldots, x_{n} \in \mathbb{R}^{d},\left|x_{i}\right|=1$, the average of $\prod\left(x_{i}, \xi\right)^{2}$ on the unit sphere $\left\{\xi \in \mathbb{R}^{d}:|\xi|=1\right\}$ is at least

$$
\frac{\Gamma(d / 2)}{2^{n} \Gamma(d / 2+n)}=\frac{(d-2) ! !}{(d+2 n-2) ! !}=\frac{1}{d(d+2)(d+4) \ldots(d+2 n-2)},
$$

with equality if and only if the vectors $x_{i}$ are pairwise orthogonal.

Proof. The average on the unit sphere is the constant in the theorem times the expectation w.r.t. the standard Gaussian measure (see e.g. [B1]). By Theorem 2.1, the latter expectation is minimal if and only if the $x_{i}$ are pairwise orthogonal, in which case it is 1 . 
Theorem 2.3. For real linear functionals $f_{i}$ on a real Euclidean space,

$$
\left\|f_{1} \cdots f_{n}\right\| \geq \frac{\left\|f_{1}\right\| \cdots\left\|f_{n}\right\|}{\sqrt{n(n+2)(n+4) \cdots(3 n-2)}} .
$$

Here $\|\cdot\|$ means supremum of the absolute value on the unit sphere. In the infinitedimensional case, functionals with infinite norm may be allowed. Then the convention $0 \cdot \infty=0$ should be used on the right hand side.

Proof. We may assume that the space is $\mathbb{R}^{d}$ with $d \leq n$, and the functionals are given by $f_{i}(\xi)=\left(x_{i}, \xi\right)$ with $\left\|f_{i}\right\|=\left|x_{i}\right|=1$. Then $\left\|f_{1} \cdots f_{n}\right\|^{2}$ is at least the average of $\prod f_{i}^{2}(\xi)=\prod\left(x_{i}, \xi\right)^{2}$ on the unit sphere, which by Theorem 2.2 and $d \leq n$ is at least $1 /(n(n+2)(n+4) \cdots(3 n-2))$.

It is an unsolved problem, raised by Benítez, Sarantopoulos and Tonge [BST] (1998), whether Theorem 2.3 is true with $n^{n}$ under the square root sign in the denominator on the right hand side. This is called the 'real linear polarization constant' problem. In the complex case, the affirmative answer was proved by Arias-de-Reyna [A] in 1998, based on the complex analog of the Wick formula [A, B2, G] and on Lieb's inequality (3). ${ }^{1}$ Keith Ball [Ball] gave another proof of the affirmative answer in the complex case by solving the complex plank problem.

In the real case, the affirmative answer for $n \leq 5$ was proved by Pappas and Révész $[\mathrm{PR}]$ in 2004. For general $n$, the best estimate known before the present paper was that of Révész and Sarantopoulos [RS] (2004), based on results of [MST], with $(2 n)^{n} / 4$ under the square root sign. See [Mat1, Mat2, MM, R] for accounts on this and related questions. Note that

$$
\begin{array}{r}
n(n+2)(n+4) \cdots(3 n-2)= \\
=\exp (\log n+\log (n+2)+\log (n+4)+\cdots+\log (3 n-2))< \\
<\exp \left(\frac{1}{2} \int_{n}^{3 n} \log u \cdot \mathrm{d} u\right)= \\
=\exp \left([u(\log u-1)]_{n}^{3 n} / 2\right)=\exp ((3 n \log 3 n-3 n-n \log n+n) / 2)= \\
=\exp \frac{n(2 \log n+3 \log 3-2)}{2}=\left(\frac{3 \sqrt{3}}{e} n\right)^{n},
\end{array}
$$

and $3 \sqrt{3} / e<3 \cdot 1.8 / 2.7=2$, so Theorem 2.3 is an improvement. Note also that the statement with $n^{n}$ under the square root sign would follow from Conjecture 1.5.

\section{Acknowledgements}

I am grateful to Péter Major, Máté Matolcsi and Szilárd Révész for helpful discussions, and to the anonymous referee for useful comments.

\footnotetext{
${ }^{1}$ The referee of the present paper called my attention to the fact that Arias-de-Reyna used only the special case of (3) where the matrix $A^{\prime}$ is of rank 1. This is much simpler than (3) in general, it can be proved essentially by the argument Marcus used in [Mar1, Mar2] to prove the even more special case $n^{\prime}=1$, which still implies inequality (2).
} 


\section{References}

[A] J. Arias-de-Reyna, Gaussian variables, polynomials and permanents, Lin. Alg. Appl. 285 (1998), 107-114.

[Ball] K. M. Ball, The complex plank problem, Bull. London. Math. Soc. 33 (2001), 433-442.

[B1] A. Barvinok, Estimating $L^{\infty}$ norms by $L^{2 k}$ norms for functions on orbits, Found. Comput. Math. 2 (2002), 393-412.

[B2] _ Integration and optimization of multivariate polynomials by restriction onto a random subspace, Found. Comput. Math. 7 (2007), no. 2, 229-244.

[BST] C. Benítez, Y. Sarantopoulos, and A. Tonge, Lower bounds for norms of products of polynomials, Math. Proc. Camb. Phil. Soc. 124 (1998), 395-408.

[D] D. Ž. Đoković, Simple proof of a theorem on permanents, Glasgow Math. J. 10 (1969), 52-54.

[G] L. Gurvits, Classical complexity and quantum entanglement, J. Comput. System Sci. 69 (2004), no. 3, 448-484.

[L] E. H. Lieb, Proofs of some conjectures on permanents, J. Math. Mech. 16 (1966), 127-134.

[Mar1] M. Marcus, The permanent analogue of the Hadamard determinant theorem, Bull. Amer. Math. Soc. 69 (1963), 494-496.

[Mar2] _ The Hadamard theorem for permanents, Proc. Amer. Math. Soc. 15 (1964), 967-973.

[MN] M. Marcus and M. Newman, The permanent function as an inner product, Bull. Amer. Math. Soc. 67 (1961), 223-224.

[Mat1] M. Matolcsi, A geometric estimate on the norm of product of functionals, Lin. Alg. Appl. 405 (2005), 304-310.

[Mat2] _ , The linear polarization constant of $\mathbb{R}^{n}$, Acta Math. Hungar. 108 (2005), no. 1-2, 129-136.

[MM] M. Matolcsi and G. A. Muñoz, On the real linear polarization constant problem, Math. Inequal. Appl. 9 (2006), no. 3, 485-494.

[Mi] H. Minc, Permanents, Encyclopedia of Mathematics and its Applications, Addison-Wesley, 1978.

[MST] G. A. Muñoz, Y. Sarantopoulos, and A. Tonge, Complexifications of real Banach spaces, polynomials and multilinear maps, Studia Math. 134 (1999), no. 1, 1-33.

[PR] A. Pappas and Sz. Révész, Linear polarization constants..., J. Math. Anal. Appl. 300 (2004), $129-146$.

[R] Sz. Gy. Révész, Inequalities for multivariate polynomials, Annals of the Marie Curie Fellowships, 4 (2006), http://www.mariecurie.org/annals/, arXiv preprint: math.CA/0703387

[RS] Sz. Gy. Révész and Y. Sarantopoulos, Plank problems, polarization and Chebyshev constants, J. Korean Math. Soc. 41 (2004) 157-174.

[S] B. Simon, The $P(\phi)_{2}$ Euclidean (Quantum) Field Theory, Princeton Series in Physics, Princeton University Press, 1974

[Z] A. Zvonkin, Matrix integrals and map enumeration: an accesible introduction, Combinatorics and physics (Marseille, 1995), Math. Comput. Modelling, 26 (1997), 281-304.

Alfréd Rényi Institute of Mathematics, Hungarian Academy of Sciences, P.O.B. 127, 1364 BudAPEST, HUNGARY

E-mail address: frenkelp@renyi.hu 